\title{
Multigrid Solution of Monotone Second-Order Discretizations of Hyperbolic Conservation Laws
}

\author{
By Stefan Spekreijse
}

\begin{abstract}
This paper is concerned with two subjects: the construction of second-order accurate monotone upwind schemes for hyperbolic conservation laws and the multigrid solution of the resulting discrete steady-state equations. By the use of an appropriate definition of monotonicity, it is shown that there is no conflict between second-order accuracy and monotonicity (neither in one nor in more dimensions).

It is shown that a symmetric block Gauss-Seidel underrelaxation (each block is associated with 4 cells) has satisfactory smoothing rates. The success of this relaxation is due to the fact that, by coupling the unknowns in such blocks, the nine-point stencil of a second-order 2D upwind discretization changes into a five-point block stencil.
\end{abstract}

1. Introduction. To obtain solutions of first-order finite-volume upwind schemes for the 2D steady Euler equations, nested nonlinear multigrid (FMG-FAS) iteration has proved to be a very efficient solution process. [6], [7]. Encouraged by this successful application of nonlinear multigrid, it is natural to ask whether it is possible to use nonlinear multigrid for the efficient solution of second-order finite-volume monotone upwind schemes as well.

To answer this question, we have to discuss the following subjects: how to construct a second-order montone upwind scheme and how to choose the nonlinear multigrid components such as the relaxation method, the restriction and prolongation operators, and the coarse grid operators.

Because of the complexity of the Euler equations (a hyperbolic system of conservation laws), we start analyzing these subjects for the less complicated scalar hyperbolic conservation laws. Scalar hyperbolic conservation laws are interesting by themselves and, without the complexity of hyperbolic systems, the analysis is more complete and more transparent. The results of the scalar analysis can be generalized, in a straightforward manner, to systems of hyperbolic conservation laws such as the Euler equations. We will report on this in a separate paper.

In Section 2 we describe the construction of second-order monotone upwind schemes. By using a definition of monotonicity based on positivity of coefficients, it is shown that there is no contradiction between monotonicity and second-order accuracy (neither in one nor in more dimensions). We emphasize that the concept of monotone schemes used in this paper is not equivalent with the definition of

Received June 4, 1986; revised October 13, 1986.

1980 Mathematics Subject Classification (1985 Revision). Primary 35L65, 65N05, 76G15.

Key words and phrases. Conservation laws, multigrid methods.

1987 American Mathematical Society $0025-5718 / 87 \$ 1.00+\$ .25$ per page 
monotone schemes by Harten, Hyman, and Lax [4]. It can easily be seen that the constructed schemes are TVD (Total Variation Diminishing) [5] in one dimension but not in two or more dimensions. This agrees with the result of Goodman and LeVeque which states that TVD schemes in 2-dimensions are at most first-order accurate [3]. Much attention is payed to the construction of a limiter. Because of its smoothness, the van Albada limiter [12] appears to be the most attractive one.

In Section 3 we show what nonlinear multigrid method we apply. In the multigrid method, a good relaxation process is of crucial importance. A block Gauss-Seidel underrelaxation (with $\omega=0.5$ ) appears to be a satisfactory smoothing operator.

In Section 4 numerical results are shown. Excellent steady solutions are obtained for linear problems with contact discontinuities and for nonlinear problems with shocks. As in the first-order case, multigrid appears to be an efficient solution process.

In the last section some conclusions are listed.

2. The Construction of a Second-Order Monotone Upwind Scheme. Consider the following nonlinear scalar hyperbolic conservation law

$$
\frac{\partial}{\partial t} u+\frac{\partial}{\partial x} f(u)+\frac{\partial}{\partial y} g(u)=0 .
$$

Suppose that the flux functions $f(u)$ and $g(u)$ can be split in positive and negative parts, i.e.,

$$
f(u)=f^{+}(u)+f^{-}(u), \quad g(u)=g^{+}(u)+g^{-}(u),
$$

where

$$
\begin{array}{cc}
\frac{d}{d u} f^{+}(u) \geqslant 0, \quad \frac{d}{d u} f^{-}(u) \leqslant 0 & \forall u \in \mathbb{R}, \\
\frac{d}{d u} g^{+}(u) \geqslant 0, & \frac{d}{d u} g^{-}(u) \leqslant 0 \quad \forall u \in \mathbb{R} .
\end{array}
$$

To discretize (2.1), we apply the finite-volume technique. Thus, the discrete values of $u$ are associated with cell centers and are regarded as approximations of the mean value of $u$ in each cell. To avoid technical details, we discretize (2.1) on an equidistant grid with mesh size $h$. Furthermore, the space discretization is based on the Projection-Evolution approach [14], [15]. Because we are only interested in steady-state solutions of (2.1), the simplest time discretization is used, i.e., "forward Euler". (Later, the time dependency in the discretized form of (2.1) is dropped, and multigrid is used to solve the nonlinear time-independent system of discretized equations directly.) Hence, (2.1) is discretized by

$$
\begin{aligned}
& U_{i, j}^{n+1}= U_{i, j}^{n}+\frac{\Delta t}{h}\left[\left\{f^{+}\left(U_{i-1 / 2, j}^{-n}\right)-f^{+}\left(U_{i+1 / 2, j}^{-n}\right)\right\}\right. \\
&\left.+\left\{f^{-}\left(U_{i-1 / 2, j}^{+n}\right)-f^{-}\left(U_{i+1 / 2, j}^{+n}\right)\right\}\right] \\
&+\frac{\Delta t}{h}\left[\left\{g^{+}\left(U_{i, j-1 / 2}^{-n}\right)-g^{+}\left(U_{i, j+1 / 2}^{-n}\right)\right\}\right. \\
&+ \\
&\left.+\left\{g^{-}\left(U_{i, j-1 / 2}^{+n}\right)-g^{-}\left(U_{i, j+1 / 2}^{+n}\right)\right\}\right],
\end{aligned}
$$


where

$$
\begin{aligned}
& U_{i+1 / 2, j}^{-n}=U_{i, j}^{n}+\frac{1}{2} \psi\left(R_{i, j}^{n}\right)\left(U_{i, j}^{n}-U_{i-1, j}^{n}\right), \\
& U_{i-1 / 2, j}^{+n}=U_{i, j}^{n}+\frac{1}{2} \psi\left(\frac{1}{R_{i, j}^{n}}\right)\left(U_{i, j}^{n}-U_{i+1, j}^{n}\right), \\
& U_{i, j+1 / 2}^{-n}=U_{i, j}^{n}+\frac{1}{2} \psi\left(S_{i, j}^{n}\right)\left(U_{i, j}^{n}-U_{i, j-1}^{n}\right), \\
& U_{i, j-1 / 2}^{+n}=U_{i, j}^{n}+\frac{1}{2} \psi\left(\frac{1}{S_{i, j}^{n}}\right)\left(U_{i, j}^{n}-U_{i, j+1}^{n}\right),
\end{aligned}
$$

and

$$
R_{i, j}^{n}=\frac{U_{i+1, j}^{n}-U_{i, j}^{n}}{U_{i, j}^{n}-U_{i-1, j}^{n}}, \quad S_{i, j}^{n}=\frac{U_{i, j+1}^{n}-U_{i, j}^{n}}{U_{i, j}^{n}-U_{i, j-1}^{n}}
$$

and $\psi: \mathbb{R} \mapsto \mathbb{R}$ is a continuous function called the limiter. The value $U_{i, j}^{n}$ is a numerical approximation of the mean value of $u$ in cell $(i, j)$ at time $t=n \Delta t$, so

$$
U_{i, j}^{n} \approx \frac{1}{h^{2}} \int_{(i-1 / 2) h}^{(i+1 / 2) h} \int_{(j-1 / 2) h}^{(j+1 / 2) h} U(\xi, \eta, n \Delta t) d \xi d \eta
$$

The values $U_{i+1 / 2, j}^{-n}, U_{i+1 / 2, j}^{+n}$ are approximations of

$$
\frac{1}{h} \int_{(j-1 / 2) h}^{(j+1 / 2) h} u\left(\left(i+\frac{1}{2}\right) h, \eta, n \Delta t\right) d \eta,
$$

located at the left and right side of the cell wall $(i+1 / 2, j)$. See Figure 2.1.

The limiter $\psi=\psi(R)$ is introduced in the discretization in order to construct a monotone, spatially second-order scheme. The limiter is a function of the consecutive gradients, a common practice in this field [2], [11], [13]. Notice that in (2.5) $\psi \equiv 0$ corresponds to the first-order upwind scheme while $\psi \equiv 1$ yields the fully one-sided second-order upwind scheme. We define a monotone scheme as follows.

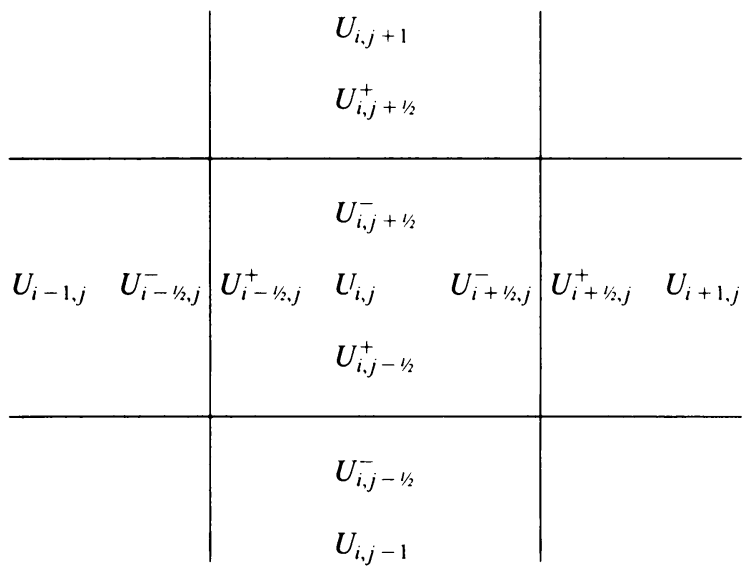

Figure 2.1

Location of the several variables in the space discretization. 
Definition. Consider a discretization of (2.1) given by

$$
\begin{aligned}
U_{i, j}^{n+1}= & U_{i, j}^{n}+A_{i+1 / 2, j}^{n}\left(U_{i+1, j}^{n}-U_{i, j}^{n}\right)+B_{i, j+1 / 2}^{n}\left(U_{i, j+1}^{n}-U_{i, j}^{n}\right) \\
& +C_{i-1 / 2, j}^{n}\left(U_{i-1, j}^{n}-U_{i, j}^{n}\right)+D_{i, j-1 / 2}^{n}\left(U_{i, j-1}^{n}-U_{i, j}^{n}\right),
\end{aligned}
$$

where

$$
\begin{aligned}
& A_{i+1 / 2, j}^{n}=A\left(\ldots, U_{i-1, j}^{n}, U_{i, j}^{n}, U_{i+1, j}^{n}, \ldots\right), \\
& B_{i, j+1 / 2}^{n}=B\left(\ldots, U_{i, j-1}^{n} U_{i, j}^{n}, U_{i, j+1}^{n}, \ldots\right), \\
& C_{i-1 / 2, j}^{n}=C\left(\ldots, U_{i-1, j}^{n}, U_{i, j}^{n}, U_{i+1, j}^{n}, \ldots\right), \\
& D_{i, j-1 / 2}^{n}=D\left(\ldots, U_{i, j-1}^{n}, U_{i, j}^{n}, U_{i, j+1}^{n}, \ldots\right) .
\end{aligned}
$$

Scheme (2.8) is called monotone if

$$
A_{i+1 / 2, j}^{n} \geqslant 0, \quad B_{i, j+1 / 2}^{n} \geqslant 0, \quad C_{i-1 / 2, j}^{n} \geqslant 0, \quad D_{i, j-1 / 2}^{n} \geqslant 0,
$$

and if

$$
1-A_{i+1 / 2, j}^{n}-B_{i, j+1 / 2}^{n}-C_{i-1 / 2, j}^{n}-D_{i, j-1 / 2}^{n} \geqslant 0 .
$$

This definition of monotonicity is especially useful for the steady-state problem, as is shown by the following theorem.

THEOREM 2.1. If scheme (2.8) is monotone, then a steady-state solution of (2.8) is monotone, i.e.,

$$
\min \left(U_{i-1, j}, U_{i+1, j}, U_{i, j-1}, U_{i, j+1}\right) \leqslant U_{i, j} \leqslant \max \left(U_{i-1, j}, U_{i+1, j}, U_{i, j-1}, U_{i}\right),
$$

where $\left\{U_{i, j}\right\}$ denotes a steady-state solution of (2.8).

Proof. From (2.8) we see that

$$
U_{i, j}=\frac{A_{i+1 / 2, j} U_{i+1, j}+B_{i, j+1 / 2} U_{i, j+1}+C_{i-1 / 2, j} U_{i-1, j}+D_{i, j-1 / 2} U_{i, j-1}}{A_{i+1 / 2, j}+B_{i, j+1 / 2}+C_{i-1 / 2, j}+D_{i, j-1 / 2}},
$$

which, owing to the positivity of the coefficients, proves the theorem immediately.

We wish to show under what conditions scheme (2.4) is monotone. It can easily be seen that scheme (2.4) can be written as (2.8) by taking

$$
\begin{aligned}
& A_{i+1 / 2, j}^{n}=-\frac{\Delta t}{h} \cdot \frac{f^{-}\left(U_{i+1 / 2, j}^{+n}\right)-f^{-}\left(U_{i-1 / 2, j}^{+n}\right)}{U_{i+1 / 2, j}^{+n}-U_{i-1 / 2, j}^{+n}} \cdot \frac{U_{i+1 / 2, j}^{+n}-U_{i-1 / 2, j}^{+n}}{U_{i+1, j}^{n}-U_{i, j}^{n}}, \\
& C_{i-1 / 2, j}^{n}=+\frac{\Delta t}{h} \cdot \frac{f^{+}\left(U_{i+1 / 2, j}^{-n}\right)-f^{+}\left(U_{i-1 / 2, j}^{-n}\right)}{U_{i+1 / 2, j}^{-n}-U_{i-1 / 2, j}^{-n}} \cdot \frac{U_{i+1 / 2, j}^{-n}-U_{i-1 / 2, j}^{-n}}{U_{i, j}^{n}-U_{i-1, j}^{n}} \\
& B_{i, j+1 / 2}^{+}=-\frac{\Delta t}{h} \cdot \frac{g^{-}\left(U_{i, j+1 / 2}^{+n}\right)-g^{-}\left(U_{i, j-1 / 2}^{+n}\right)}{U_{i, j+1 / 2}^{+n}-U_{i, j-1 / 2}^{+n}} \cdot \frac{U_{i, j+1 / 2}^{+n}-U_{i, j-1 / 2}^{+n}}{U_{i, j+1}^{n}-U_{i, j}^{n}}, \\
& D_{i, j-1 / 2}^{n}=+\frac{\Delta t}{h} \cdot \frac{g^{+}\left(U_{i, j+1 / 2}^{-n}\right)-g^{+}\left(U_{i, j-1 / 2}^{-n}\right)}{U_{i, j+1 / 2}^{-n}-U_{i, j-1 / 2}^{-n}} \cdot \frac{U_{i, j+1 / 2}^{-n}-U_{i, j-1 / 2}^{-n}}{U_{i, j}^{n}-U_{i, j-1}^{n}}
\end{aligned}
$$


To obtain positivity of the coefficients $A_{i+1 / 2, j}^{n}, B_{i, j+1 / 2}^{n}$, etc., it is sufficient (by the Mean Value Theorem) that

$$
\begin{array}{ll}
\frac{U_{i+1 / 2, j}^{+n}-U_{i-1 / 2, j}^{+n}}{U_{i+1, j}^{n}-U_{i, j}^{n}} \geqslant 0, & \frac{U_{i+1 / 2, j}^{-n}-U_{i-1 / 2, j}^{-n}}{U_{i, j}^{n}-U_{i-1, j}^{n}} \geqslant 0, \\
\frac{U_{i, j+1 / 2}^{+n}-U_{i, j-1 / 2}^{+n}}{U_{i, j+1}^{n}-U_{i, j}^{n}} \geqslant 0, & \frac{U_{i, j+1 / 2}^{-n}-U_{i, j-1 / 2}^{-n}}{U_{i, j}^{n}-U_{i, j-1}^{n}} \geqslant 0 .
\end{array}
$$

Furthermore, inequality $(2.10 \mathrm{~b})$ is fulfilled by taking $\Delta t$ sufficiently small, while assuming uniform boundedness of the derivatives of $f^{+}(u), f^{-}(u), g^{+}(u)$ and $g^{-}(u)$, and taking care that the left-hand sides of the inequalities in (2.12) are also uniformly bounded.

By substitution of (2.5) in (2.12) it is easily seen that (2.12) is fulfilled if

$$
1+\frac{1}{2} \psi(R)-\frac{1}{2} \psi(S) \cdot \frac{1}{S} \geqslant 0 \quad \forall R, S \in \mathbb{R} .
$$

Furthermore, the uniform boundedness of the left-hand side of the inequalities in (2.12) is obtained by requiring

$$
\psi(R)-\psi(S) \cdot \frac{1}{S} \leqslant 2 M \quad \forall R, S \in \mathbb{R}, M \in(0, \infty) .
$$

So, (2.1) is a monotone scheme if the limiter $\psi=\psi(R)$ satisfies the property that

$$
-2 \leqslant \psi(R)-\psi(S) \cdot \frac{1}{S} \leqslant 2 M \quad \forall R, S \in \mathbb{R} .
$$

This inequality is satisfied if

$$
\alpha \leqslant \psi(R) \leqslant M \quad \forall R \in \mathbb{R}
$$

and

$$
-M \leqslant \frac{\psi(R)}{R} \leqslant 2+\alpha \quad \forall R \in \mathbb{R} .
$$

The monotonicity region given by (2.16) is depicted in Figure 2.2. We assume $\alpha \in[-2,0]$.

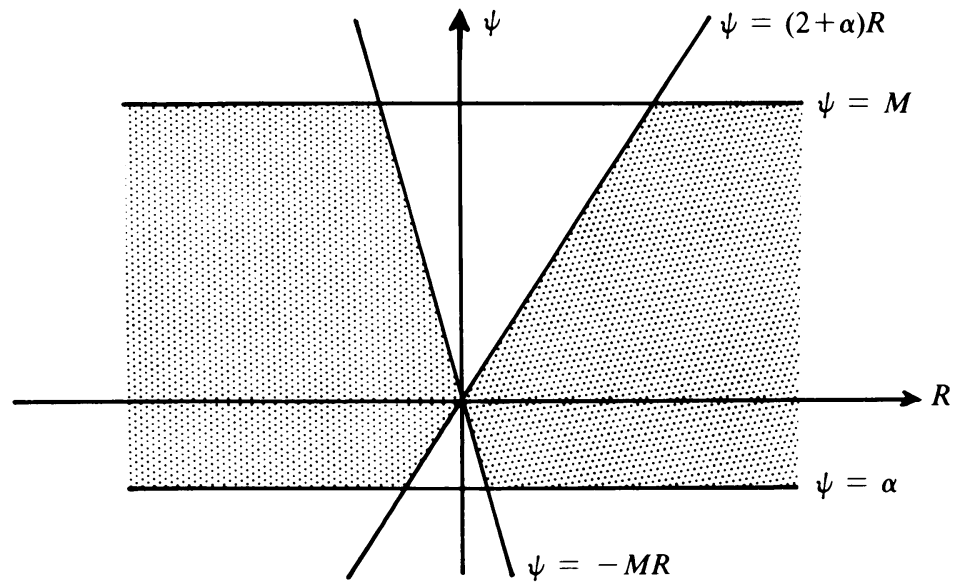

FIGURE 2.2

Monotonicity region. 
We have thus found the following theorem.

THEOREM 2.2. If the limiter $\psi=\psi(R)$ has the properties that there exist constants $M \in(0, \infty), \alpha \in[-2,0]$ such that $\alpha \leqslant \psi(R) \leqslant M,-M \leqslant \psi(R) / R \leqslant 2+\alpha$ for all $R \in \mathbb{R}$, then (2.4) is a monotone scheme.

One of the direct consequences of Theorem 2.2 is that $\psi(0)=0$. Notice that $\psi \equiv 0$, which corresponds to the first-order upwind scheme, results in a monotone scheme, as we should expect.

Now, we wish to investigate under which condition scheme (2.4) is second-order accurate with respect to the space discretization. Define

$$
\begin{aligned}
& \tilde{U}_{i+1 / 2, j}^{-}=U_{i, j}+\frac{1}{2}\left(U_{i, j}-U_{i-1, j}\right), \\
& U_{i+1 / 2, j}^{-}=U_{i, j}+\frac{1}{2} \psi\left(R_{i, j}\right)\left(U_{i, j}-U_{i-1, j}\right), \\
& \tilde{U}_{i-1 / 2, j}^{+}=U_{i, j}+\frac{1}{2}\left(U_{i, j}-U_{i+1, j}\right), \\
& U_{i-1 / 2, j}^{+}=U_{i, j}+\frac{1}{2} \psi\left(\frac{1}{R_{i, j}}\right)\left(U_{i, j}-U_{i+1, j}\right),
\end{aligned}
$$

with similar formulas for $\tilde{U}_{i, j \pm 1 / 2}^{ \pm}$and $U_{i, j \pm 1 / 2}^{ \pm}$. Notice that the $\tilde{U}$-values correspond to $\psi(R) \equiv 1$, the fully one-sided upwind case, which gives a second-order accurate space discretization.

LEMMA 2.1. If the limiter $\psi=\psi(R)$ is constructed such that

$$
U_{i+1 / 2, j}^{-}-U_{i-1 / 2, j}^{-}=\tilde{U}_{i+1 / 2, j}^{-}-\tilde{U}_{i-1 / 2, j}^{-}+O\left(h^{3}\right)
$$

and

$$
U_{i+1 / 2, j}^{-}=\tilde{U}_{i+1 / 2, j}^{-}+O\left(h^{2}\right),
$$

where $U_{i+1 / 2, j}^{-}, \tilde{U}_{i+1 / 2, j}^{-}$etc. are given by (2.17), then (2.4) is second-order accurate with respect to the space discretization.

Proof. This lemma is a direct consequence of the formulas (2.30) and (2.31) derived in [10].

From (2.17) we see that

$$
U_{i+1 / 2, j}^{-}=\tilde{U}_{i+1 / 2, j}^{-}+\frac{1}{2}\left(\psi\left(R_{i, j}\right)-1\right) \cdot\left(U_{i, j}-U_{i-1, j}\right) .
$$

Furthermore, by assuming that $\partial u / \partial x$ is bounded away from 0 , we see that

$$
R_{i, j}=\frac{U_{i+1, j}-U_{i, j}}{U_{i, j}-U_{i-1, j}}=1+\frac{U_{i+1, j}-2 U_{i, j}+U_{i-1, j}}{U_{i, j}-U_{i-1, j}}=1+O(h) .
$$

Hence, using (2.20), we can write

$$
\psi\left(R_{i, j}\right)=\psi(1)+\frac{d \psi}{d R}(1) \cdot\left(R_{i, j}-1\right)+O\left(h^{2}\right)
$$


where we assume that $\psi(R)$ is twice continuously differentiable in a neighborhood of $R=1$. So, if $\psi(1)=1$, we immediately see from (2.19)-(2.21) that (2.18b) holds. Furthermore, if $\psi(1)=1$, then also

$$
\begin{aligned}
U_{i+1 / 2, j}^{-}= & \tilde{U}_{i+1 / 2, j}^{-}+\frac{1}{2} \frac{d \psi}{d R}(1) \cdot\left(\frac{U_{i+1, j}-2 U_{i, j}+U_{i-1, j}}{U_{i, j}-U_{i-1, j}}\right) \\
& \cdot\left(U_{i, j}-U_{i-1, j}\right)+O\left(h^{3}\right) \\
= & \tilde{U}_{i+1 / 2, j}^{-}+\frac{1}{2} \frac{d \psi}{d R}(1)\left(U_{i+1, j}-2 U_{i, j}+U_{i-1, j}\right)+O\left(h^{3}\right) .
\end{aligned}
$$

From (2.22) it is easily seen that also (2.18a) holds. Therefore we may conclude that $\psi(1)=1$ is a sufficient condition to obtain a second-order space discretization.

THEOREM 2.3. If $\psi(1)=1$ and if $\psi \in C^{2}$ in a neighborhood of 1 , then scheme (2.4) is second-order accurate with respect to the space discretization.

Corollary 2.1. Scheme (2.4) is linear if $\psi(R)=a+b R, a, b \in \mathbb{R}$. From Theorems 2.2 and 2.3 it is easily seen that no linear schemes exist that combine the property of second-order accuracy and monotonicity.

Examples of limiters combining the property of second-order accuracy and monotonicity are:

Example 1. The van Leer limiter [11], [13], [14]

$$
\psi_{\mathrm{VL}}(R)=\frac{R+|R|}{|R|+1}
$$

By taking $M=2$ and $\alpha=0$ it is easily seen that this limiter satisfies the monotonicity restriction (2.16). Because $\psi_{\mathrm{VL}}(1)=1$, second-order accuracy is obtained.

Example 2. The van Albada limiter [12]

$$
\psi_{\mathrm{VA}}(R)=\frac{R^{2}+R}{R^{2}+1} .
$$

By taking $M=2$ and $\alpha=-\frac{1}{2}$, it is easily seen that this limiter combines monotonicity with second-order accuracy. Another advantage of this limiter is that $\psi_{\mathrm{vA}} \in$ $C^{\infty}(\mathbb{R})$. This is an important property when we apply Newton's method (local linearization) in a relaxation procedure for the solution of the steady-state discrete equations.

For a review of other limiters, see [11]. But notice that a limiter $\phi(r)$ of [11] is related to $\psi(R)$ by $R=1 / r, \psi(R)=R \phi(r)$. A limiter $\phi(r)$ of [11] is only algebraically identical with $\psi(R)$ if $\psi(R) / R=\psi(1 / R)$. For our numerical experiments in Section 4 we have chosen van Albada's limiter because of its smoothness.

Remark 2.1. It has been observed [9], [14] that second-order accuracy can be achieved by assuming a linear distribution in each cell, rather than the uniform distribution associated with first-order schemes. In a cell, a linear distribution in the $x$-direction is achieved if

$$
U_{i+1 / 2, j}^{-}-U_{i, j}=U_{i, j}-U_{i-1 / 2, j}^{+} ;
$$


similarly in the $y$-direction. Using (2.17), this means

$$
\psi\left(R_{i, j}\right)\left(U_{i, j}-U_{i-1, j}\right)=\psi\left(\frac{1}{R_{i, j}}\right)\left(U_{i+1, j}-U_{i, j}\right)
$$

or, equivalently,

$$
\psi\left(\frac{1}{R_{i, j}}\right)=\frac{\psi\left(R_{i, j}\right)}{R_{i, j}}
$$

Thus, if a limiter satisfies

$$
\psi\left(\frac{1}{R}\right)=\frac{\psi(R)}{R} \quad \forall R \in \mathbb{R},
$$

we can speak of linear distributions in each cell. It can be verified that both $\psi_{\mathrm{VL}}$ and $\psi_{\mathrm{VA}}$ possess this property. This is no coincidence: They were designed that way. Notice that if a limiter $\psi=\psi(R)$ satisfies (2.25), then the monotonicity conditions (2.16) are equivalent to

$$
\alpha \leqslant \psi(R) \leqslant M, \quad-M \leqslant \psi(R) \leqslant 2+\alpha \quad \forall R \in \mathbb{R} .
$$

Formula (2.25) implies $\psi(0)=0$, hence $\alpha \in[-2,0]$. By taking $M=2,(2.26)$ becomes

$$
\alpha \leqslant \psi(R) \leqslant 2+\alpha \quad \forall R \in \mathbb{R}
$$

which means

$$
\psi_{\max }-\psi_{\min } \leqslant 2
$$

where

$$
\psi_{\max }=\max _{R \in \mathbb{R}}(\psi(R)), \quad \psi_{\min }=\min _{R \in \mathbb{P}}(\psi(R)) .
$$

Hence, we have found the following result.

THEOREM 2.4. If a limiter $\psi=\psi(R)$ has the property $\psi(1 / R)=\psi(R) / R$, then scheme (2.4) is monotone if $\psi_{\max }-\psi_{\min } \leqslant 2$.

Remark 2.2. We will use Newton's method (local linearization) in the relaxation. Therefore, we have to linearize the limiter. It can easily be verified that

$$
U_{i+1 / 2}=U_{i}-\frac{1}{2} \psi\left(R_{i}\right)\left(U_{i}-U_{i-1}\right)
$$

with

$$
R_{i}=\frac{U_{i+1}-U_{i}}{U_{i}-U_{i-1}}
$$

implies

$$
\begin{aligned}
& \partial U_{i+1 / 2}=\alpha\left(R_{i}\right) \partial U_{i-1} \\
& \partial U_{i+1 / 2}=\beta\left(R_{i}\right) \partial U_{i}, \\
& \partial U_{i+1 / 2}=\gamma\left(R_{i}\right) \partial U_{i+1}
\end{aligned}
$$


where

$$
\begin{aligned}
& \alpha(R)=-\frac{1}{2} \psi(R)+\frac{1}{2} R \frac{d \psi}{d R}(R), \\
& \beta(R)=1+\frac{1}{2} \psi(R)-\frac{1}{2}(1+R) \frac{d \psi}{d R}(R), \\
& \gamma(R)=\frac{1}{2} \frac{d \psi}{d R}(R) .
\end{aligned}
$$

So we see that the derivative of $U_{i+1 / 2}$ with respect to $U_{i-1}, U_{i}$ or $U_{i+1}$ only depends on $R_{i}$ !

Remark 2.3. To avoid any confusion, we wish to emphasize that in this article the monotonicity is obtained by multiplying the backward differences with $\psi(R)$, i.e.,

$$
\begin{aligned}
& U_{i+1 / 2, j}^{-}=U_{i, j}+\frac{1}{2} \psi\left(R_{i, j}\right)\left(U_{i, j}-U_{i-1, j}\right), \\
& U_{i-1 / 2, j}^{+}=U_{i, j}+\frac{1}{2} \psi\left(\frac{1}{R_{i, j}}\right)\left(U_{i, j}-U_{i+1, j}\right) .
\end{aligned}
$$

Another possibility, often encountered in the literature, is

$$
\begin{aligned}
& U_{i+1 / 2, j}^{-}=U_{i, j}+\frac{1}{2} \phi\left(R_{i, j}\right)\left(\frac{U_{i+1, j}-U_{i, j}}{2}+\frac{U_{i, j}-U_{i-1, j}}{2}\right), \\
& U_{i-1 / 2, j}^{+}=U_{i, j}+\frac{1}{2} \phi\left(\frac{1}{R_{i, j}}\right)\left(\frac{U_{i-1, j}-U_{i, j}}{2}+\frac{U_{i, j}-U_{i+1, j}}{2}\right) .
\end{aligned}
$$

It can be verified that both descriptions are equivalent if

$$
\psi(R)=\phi(R) \cdot\left(\frac{R+1}{2}\right) .
$$

The preceding results show clearly that, from a theoretical point of view, description (2.28) is preferable to description (2.29).

3. Multigrid Solution. In this paper, as noted before, we are primarily interested in monotone second-order accurate steady-state solutions of (2.1). Therefore, we omit the superscript $n$ in (2.4)-(2.6), and we wish to solve

$$
\begin{aligned}
&\left(L_{h} u\right)_{i, j}:=h\left[\left\{f^{+}\left(u_{i+1 / 2, j}^{-}\right)-f^{+}\left(u_{i-1 / 2, j}^{-}\right)\right\}\right. \\
&+\left\{f^{-}\left(u_{i+1 / 2, j}^{+}\right)-f^{-}\left(u_{i-1 / 2, j}^{+}\right)\right\} \\
&+\left\{g^{+}\left(u_{i, j+1 / 2}^{-}\right)-g^{+}\left(u_{i, j-1 / 2}^{-}\right)\right\} \\
&\left.+\left\{g^{-}\left(u_{i, j+1 / 2}^{+}\right)-g^{-}\left(u_{i, j-1 / 2}^{+}\right)\right\}\right] \\
&=\left(r_{h}\right)_{i, j}
\end{aligned}
$$

directly. Here $r_{h} \equiv 0$ and $h$ denotes the meshsize of the finest grid. Note that in (3.1) we multiply with the meshsize $h$ instead of dividing by $h$, as was done in (2.4). By doing this, $\left(L_{h} u\right)_{i, j}$ receives the physical meaning of "net flux" into cell $(i, j)$. This is a more appropriate quantity when dealing with nonuniform grids. 
For the multigrid solution of (3.1) and the multigrid terminology used, we refer to [1]. A nonlinear (FAS) multigrid solution of (3.1) is obtained by applying, iteratively, FAS-cycles. One FAS-cycle for the solution of (3.1) consists of the following steps:

(0) Start with an approximate solution $u_{h}$.

(1) Improve $u_{h}$ by application of $p$ (pre-) relaxation iterations to $\left(L_{h} u_{h}\right)=r_{h}$.

(2) Compute the defect $d_{h}=r_{h}-L_{h} u_{h}$.

(3) Find an approximation $u_{2 h}$ of $u_{h}$ on the next coarser grid; $u_{2 h}:=\hat{I}_{h}^{2 h} u_{h}$, where $\hat{I}_{h}^{2 h}$ is a restriction operator.

(4) Compute $r_{2 h}=L_{2 h} u_{2 h}+I_{h}^{2 h} d_{h}$, where $I_{h}^{2 h}$ is (another) restriction operator and $L_{2 h}$ is the coarse-grid operator (an approximation of $L_{h}$ on the next coarser grid).

(5) Approximate the solution of

$$
L_{2 h} u_{2 h}=r_{2 h}
$$

by application of $\sigma$ FAS-cycles, starting with the initial estimate $u_{2 h}=\hat{I}_{h}^{2 h} u_{h}$. The result is called $u_{2 h}$.

(6) Correct the current solution by

where $I_{2 h}^{h}$ is a prolongation operator.

$$
u_{h}:=u_{h}+I_{2 h}^{h}\left(\tilde{u}_{2 h}-u_{2 h}\right)
$$

(7) Improve $u_{h}$ by application of $q$ (post-) relaxation iterations to $L_{h} u_{h}=r_{h}$.

The steps (2)-(6) in this process are called "coarse-grid correction". In order to complete the description of the FAS-cycle, we have to discuss

(0) the relation between the fine and coarse grid;

(1) the choice of the operators $L_{2 h}, I_{2 h}^{h}, I_{h}^{2 h}$, and $\hat{I}_{h}^{2 h}$;

(2) the relaxation method;

(3) the FAS-strategy, i.e., the numbers $p, q, \sigma(\sigma=1$ characterizes a $V$-cycle, $\sigma=2$ a $W$-cycle).

We now discuss these topics.

(0) Fine-grid construction. A finer grid is constructed from a coarser one by subdivision of a coarse-grid cell in 4 smaller cells as shown in Figure 3.1.

(1) Choice of the operators. The restriction operator $\hat{I}_{h}^{2 h}$ is defined by

$$
\begin{aligned}
\left(u_{2 h}\right)_{i, j} & =\left(\hat{I}_{h}^{2 h} u_{h}\right)_{i, j} \\
& :=\frac{1}{4}\left\{\left(u_{h}\right)_{2 i, 2 j}+\left(u_{h}\right)_{2 i-1,2 j}+\left(u_{h}\right)_{2 i, 2 j-1}+\left(u_{h}\right)_{2 i-1,2 j-1}\right\} .
\end{aligned}
$$
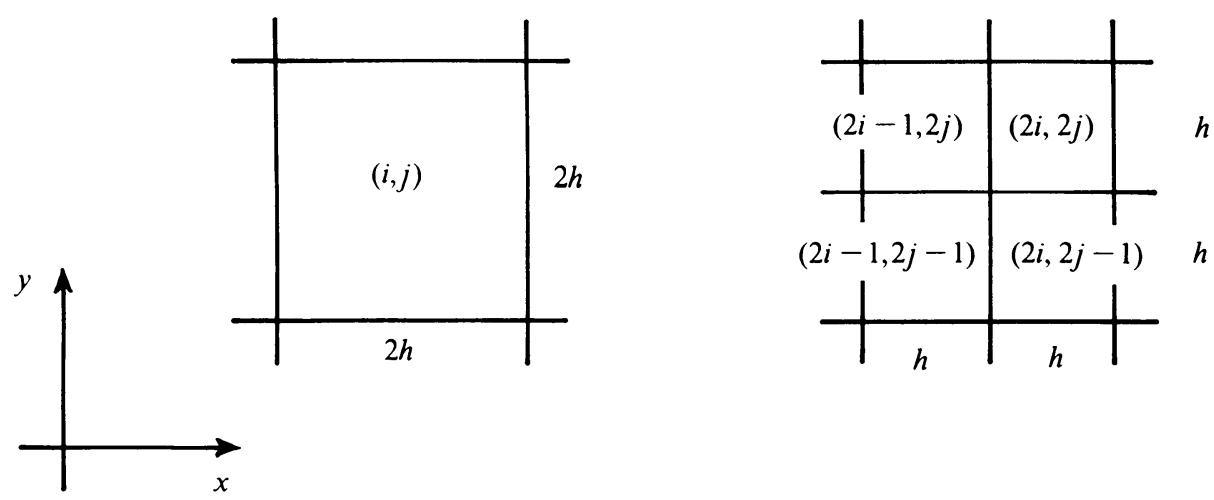

Figure 3.1

The subdivision of a coarse-grid cell in four fine-grid cells. 
The restriction operator $I_{h}^{2 h}$ is defined by

$$
\left(r_{2 h}\right)_{i, j}=\left(I_{h}^{2 h} r_{h}\right)_{i, j}=\left(r_{h}\right)_{2 i, 2 j}+\left(r_{h}\right)_{2 i-1,2 j}+\left(r_{h}\right)_{2 i, 2 j-1}+\left(r_{h}\right)_{2 i-1,2 j-1} .
$$

The prolongation operator $I_{2 h}^{h}$ is defined by

$$
\begin{aligned}
\left(I_{2 h}^{h} u_{2 h}\right)_{2 i, 2 j} & :=\left(I_{2 h}^{h} u_{2 h}\right)_{2 i-1,2 j}:=\left(I_{2 h}^{h} u_{2 h}\right)_{2 i, 2 j-1} \\
& :=\left(I_{2 h}^{h} u_{2 h}\right)_{2 i-1,2 j-1}:=\left(u_{2 h}\right)_{i, j} .
\end{aligned}
$$

The coarse-grid operator $L_{2 h}$ is defined by a Galerkin approximation

$$
L_{2 h}=I_{h}^{2 h} L_{h} I_{2 h}^{h} \text {. }
$$

Then the following theorem can be proven.

THEOREM 3.1. If the restriction and prolongation operators $I_{h}^{2 h}$ and $I_{2 h}^{h}$ are defined by (3.3) and (3.4), and if the coarse-grid operator $L_{2 h}$ is defined by the Galerkin approximation (3.5), then the coarse-grid operator corresponds to the monotone firstorder discretization $(\psi \equiv 0)$ of the continuous problem on the coarser grid. In other words,

$$
\begin{aligned}
\left(L_{2 h} u\right)_{i, j}=2 h\left[\left\{f^{+}\left(u_{i, j}\right)-f^{+}\left(u_{i-1, j}\right)\right\}+\left\{f^{-}\left(u_{i+1, j}\right)-f^{-}\left(u_{i, j}\right)\right\}\right. \\
\left.+\left\{g^{+}\left(u_{i, j}\right)-g^{+}\left(u_{i, j-1}\right)\right\}+\left\{g^{-}\left(u_{i, j+1}\right)-g^{-}\left(u_{i, j}\right)\right\}\right] .
\end{aligned}
$$

This is independent of the limiter used in (3.1)!.

Proof. The proof of this theorem is left as an exercise to the reader. We only wish to remark that for a (fine-) grid distribution $\left\{u_{h}\right\}_{i, j}$ with the property

$$
\left(u_{h}\right)_{2 i, 2 j}=\left(u_{h}\right)_{2 i-1,2 j}=\left(u_{h}\right)_{2 i, 2 j-1}=\left(u_{h}\right)_{2 i-1,2 j-1} \quad \forall(i, j)
$$

there holds

$$
\left(u_{h}\right)_{i+1 / 2, j}^{--}=\left(u_{h}\right)_{i, j+1 / 2}^{-}=\left(u_{h}\right)_{i-1 / 2, j}^{+}=\left(u_{h}\right)_{i, j-1 / 2}^{+}=\left(u_{h}\right)_{i, j} \quad \forall(i, j),
$$

where $\left(u_{h}\right)_{i+1 / 2, j}^{-},\left(u_{h}\right)_{i, j+1 / 2}^{-},\left(u_{h}\right)_{i-1 / 2, j}^{+}$and $\left(u_{h}\right)_{i, j-1 / 2}^{+}$are calculated according to (2.5), (2.6) (omitting the superscript $n$ ). This result is due to the fact that the limiter $\psi=\psi(R)$ is uniformly bounded and $\psi(0)=0$.

This theorem has an important practical consequence. We already know that nonlinear multigrid is a good solution method for the first-order upwind scheme [6], [7]. Therefore we may expect to have no problems in the solution procedure on the coarser grids.

(2) The relaxation method. As noted before, it is our purpose to apply the methods developed in this paper to systems of hyperbolic conservation laws, e.g., the Euler equations. Now, it is well known that symmetric point Gauss-Seidel relaxation is a good relaxation method in the nonlinear multigrid solution procedure for the first-order system of the steady Euler equations in 2D, but not for the second-order discrete system [6], [8]. Even for the simple scalar model problems discussed in Section 4, point relaxation methods did not work well. An explanation is that, for second-order discretizations of steady hyperbolic problems, a Gauss-Seidel point relaxation in the upstream direction causes amplification of the error (which does not happen for first-order discretizations). This is the reason why we shall investigate 
a block Gauss-Seidel relaxation, rather than a point relaxation. We shall require that no amplification of the error should occur when the block Gauss-Seidel relaxation has the upstream direction.

How do we choose the blocks? Notice that by (3.1), nine variables $u_{i, j}, u_{i+1, j}$, $u_{i+2, j}, u_{i, j+1}, u_{i, j+2}, u_{i-1, j}, u_{i-2, j}, u_{i, j-1}$, and $u_{i, j-2}$ are coupled. Therefore, we have a nine-point stencil. On the other hand, if we combine $u_{2 i, 2 j}, u_{2 i-1,2 j}, u_{2 i, 2 j-1}$, and $u_{2 i-1,2 j-1}$ to an unknown vector $U_{i, j}$ as

$$
U_{i, j}:=\left(u_{2 i, 2 j}, u_{2 i-1,2 j}, u_{2 i, 2 j-1}, u_{2 i-1,2 j-1}\right)^{T},
$$

and if we replace system (3.1) by an equivalent system with unknowns $\left\{U_{i, j}\right\}$, then we see that each equation in this new system corresponds to a five-point block stencil, i.e., $U_{i, j}$ is only coupled to $U_{i+1, j}, U_{i, j+1}, U_{i-1, j}$, and $U_{i, j-1}$. For this reason, we consider the cells $(2 i, 2 j),(2 i-1,2 j),(2 i, 2 j-1)$, and $(2 i-1,2 j-1)$ as one block. Thus, in our block Gauss-Seidel relaxation, the blocks of unknowns are scanned in succession and for each block the corresponding equations are solved simultaneously. We use Newton's method to solve these four nonlinear equations in each block.

In the following example we use local mode analysis to investigate whether our block Gauss-Seidel relaxation amplifies the error when the blocks are scanned in the upstream direction.

Example. Consider the 1D problem

$$
\frac{\partial u}{\partial t}+a \frac{\partial u}{\partial x}=0, \quad a>0 .
$$

With the second-order discretizations as described in Section 2, the system of discrete steady-state equations becomes

$$
\begin{aligned}
\left(L_{h} u\right)_{i}=a\left[u_{i}\right. & +\frac{1}{2} \psi\left(R_{i}\right)\left(u_{i}-u_{i-1}\right) \\
& \left.-\left\{u_{i-1}+\frac{1}{2} \psi\left(R_{i-1}\right)\left(u_{i-1}-u_{i-2}\right)\right\}\right]=0 .
\end{aligned}
$$

Without a limiter $(\psi \equiv 1)$ we obtain

$$
\left(L_{h} u\right)_{i}=\alpha\left[3 u_{i}-4 u_{i-1}+u_{i-2}\right]=0 \text {, }
$$

where $\alpha=a / 2$. This system is now equivalent to

$$
\left(L_{h} U\right)_{i}=\alpha\left\{\left(\begin{array}{cc}
3 & -4 \\
0 & 3
\end{array}\right) U_{i}+\left(\begin{array}{cc}
1 & 0 \\
-4 & 1
\end{array}\right) U_{i-1}\right\}=0,
$$

where $U_{i}=\left(u_{2 i}, u_{2 i-1}\right)^{T}$.

If we apply to this new system point Gauss-Seidel relaxation (which corresponds to block Gauss-Seidel relaxation for system (3.9)) in the downstream direction, it is immediately clear that an exact solution is obtained in a single iteration sweep.

Gauss-Seidel relaxation in the upstream direction gives

$$
\alpha\left\{\left(\begin{array}{cc}
3 & -4 \\
0 & 3
\end{array}\right) U_{i}^{n+1}+\left(\begin{array}{cc}
1 & 0 \\
-4 & 1
\end{array}\right) U_{i-1}^{n}\right\}=0
$$

where $n$ is the iteration index. Suppose $U_{k}^{n}=A e^{i \theta k}, U_{k}^{n+1}=G(\theta) U_{k}^{n}$, where $\theta \in$ $[-\pi, \pi]$ and $A$ is an arbitrary vector with 2 components and $G(\theta)$ a $2 \times 2$ matrix. From (3.12) it is seen that

$$
\left(\begin{array}{cc}
3 & -4 \\
0 & 3
\end{array}\right) G(\theta)+\left(\begin{array}{cc}
1 & 0 \\
-4 & 1
\end{array}\right) e^{-i \theta}=0
$$


and the eigenvalues of $G(\theta)$ are $\lambda_{G}^{1}(\theta)=e^{-i \theta}$ and $\lambda_{G}^{2}(\theta)=\frac{1}{9} e^{-i \theta}$. Hence the spectral radius of $G(\theta)$ is equal to 1 for all $\theta \in[-\pi, \pi]$, and the smoothing factor $\mu_{G}$, defined by

$$
\mu_{G}:=\max _{\pi / 2 \leqslant|\theta| \leqslant \pi}\left(\left|\lambda_{G}^{1}(\theta)\right|,\left|\lambda_{G}^{2}(\theta)\right|\right),
$$

is also equal to 1 . The smoothing factor $\mu_{G}$ can be improved by underrelaxation. This means that (3.11) is replaced by

$$
\begin{aligned}
& \alpha\left\{\left(\begin{array}{cc}
3 & -4 \\
0 & 3
\end{array}\right) \tilde{U}_{i}^{n+1}+\left(\begin{array}{cc}
1 & 0 \\
-4 & 1
\end{array}\right) U_{i-1}^{n}\right\}=0, \\
& U_{i}^{n+1}:=U_{i}^{n}+\omega\left(\tilde{U}_{i}^{n+1}-U_{i}^{n}\right),
\end{aligned}
$$

where $\omega \in[0,1]$. Again, assume that $U_{k}^{n}=A e^{i \theta k}$ and $U_{k}^{n+1}=G_{\omega}(\theta) U_{k}^{n}$; then

$$
G_{\omega}(\theta)=(1-\omega) I+\omega G(\theta),
$$

and the eigenvalues of $G_{\omega}(\theta)$ are $\lambda_{G_{\omega}}^{1,2}(\theta)=1-\omega+\omega \lambda_{G}^{1,2}(\theta)$. The optimal (smallest) smoothing factor $\mu_{G_{\omega}}$ is obtained by $\omega=0.5$; then

$$
\mu_{G_{\omega}}=\left|\frac{1}{2}+\frac{1}{2} i\right|=\frac{1}{\sqrt{2}} \approx 0.71 \text {. }
$$

From this example we see that $\omega=1$ and $\omega=0.5$ are optimal choices for the block Gauss-Seidel underrelaxation method in the downstream and upstream direction, respectively. Since we wish to use a problem-independent relaxation method, a fixed $\omega$ is used for all problems and all directions. In the context of the multigrid method where a single symmetric block Gauss-Seidel underrelaxation was used both in the pre- and the post-relaxation, it is shown by numerical experiments that in general $\omega=0.5$ is a better choice than $\omega=1.0$.

(3) The FAS-strategy. We take $p=q=1$. Due to the fact that the coarse-grid equations are first-order accurate (cf. Theorem 3.1), each coarse-grid equation $(\psi \equiv 0)$ corresponds to a five-point stencil. Therefore, we use a simple symmetric point Gauss-Seidel relaxation on the coarse grids, and so we can afford to apply $W$-cycle FAS-iterations (i.e., $\sigma=2$ ).

4. Numerical Results. For the numerical experiments in this section we have applied the multigrid method described in Section 3.

In case of linear problems, the first-order scheme $(\psi \equiv 0)$ is linear but, due to the nonlinear van Albada limiter, the second-order scheme is nonlinear. On the coarse grids we always deal with first-order schemes (cf. Theorem 3.1). Hence, for linear problems, the coarse-grid equations are linear. It is easily seen that in case of the following linear examples (Examples 1 and 2) a single post- and pre-relaxation (which are symmetric point Gauss-Seidel relaxations in different directions) is sufficient to solve the first-order system of discrete equations on the coarser grid exactly. Hence, in those linear cases, the coarse-grid correction is calculated exactly, and just one coarser grid is needed in the multigrid process. In those cases a $W$-cycle is superfluous; a $V$-cycle is sufficient.

After each FAS-iteration, on the finest grid, the $L_{1}$-norm of the residuals has been calculated, i.e.,

$$
\left\|L_{h} u_{h}^{n}-r_{h}\right\|_{L_{1}}=\sum_{(i, j)}\left|\left(L_{h} u_{h}^{n}\right)_{i, j}-\left(r_{h}\right)_{i, j}\right|
$$


where $L_{h}$ and $r_{h}$ are defined by (3.1), $n$ is the FAS-iteration index, $u_{h}^{n}$ is the current approximation of the steady-state solution of the second-order scheme and the pairs $(i, j)$ are the cell indices of the finest grid. After $n$ FAS-iterations we can calculate the approximate convergence factor $\rho_{n}$ according to

$$
\rho_{n}=\left(\frac{\left\|L_{h} u_{h}^{n}-r_{h}\right\|_{L_{1}}}{\left\|L_{h} u_{h}^{0}-r_{h}\right\|_{L_{1}}}\right)^{1 / n} .
$$

The initial iterate $u_{h}^{0}$ is obtained by the full multigrid method [1], [6]. For each multigrid process, the convergence factor $\rho=\lim _{n \rightarrow \infty} \rho_{n}$ is approximated from the finite set $\left\{\rho_{n}\right\}$.

Example 1 . On the square $[0,1] \times[0,1]$ we consider the linear convection problem

$$
\frac{\partial u}{\partial t}+a \frac{\partial u}{\partial x}+b \frac{\partial u}{\partial y}=0
$$

where $a=\cos \phi, b=\sin \phi, \phi \in(0, \pi / 2)$. Hence,

$$
\begin{array}{ll}
f(u)=f^{+}(u)=a u, & f^{-}(u)=0, \\
g(u)=g^{+}(u)=b u, & g^{-}(u)=0 .
\end{array}
$$

The boundary conditions (steady-state problem) are

$$
\left\{\begin{array}{ll}
u(0, y)=1, & 0<y<1 \\
u(x, 0)=0, & 0<x<1
\end{array} .\right.
$$

The exact solution of the steady-state problem is trivially

$$
\begin{array}{ll}
u_{e x}(x, y)=1 & \text { if } b x-a y<0 \\
u_{e x}(x, y)=0 & \text { if } b x-a y>0
\end{array}
$$

Thus, the exact solution contains a contact discontinuity.

The observed convergence factors of the multigrid solution process have been calculated for several angles $\phi$ and for several meshsizes. The results are summarized in Table 4.1.

TABLE 4.1

The approximate convergence factors of the multigrid process for several angles $\phi$ and for several equidistant meshes with size $h$.

\begin{tabular}{|c|c|c|c|c|c|}
\hline$h$ & $15^{\circ}$ & $30^{\circ}$ & $45^{\circ}$ & $60^{\circ}$ & $75^{\circ}$ \\
\hline $1 / 8$ & 0.11 & 0.13 & 0.13 & 0.13 & 0.11 \\
\hline $1 / 16$ & 0.17 & 0.25 & 0.27 & 0.25 & 0.17 \\
\hline $1 / 32$ & 0.29 & 0.38 & 0.39 & 0.38 & 0.29 \\
\hline $1 / 64$ & 0.41 & 0.44 & 0.47 & 0.43 & 0.42 \\
\hline
\end{tabular}




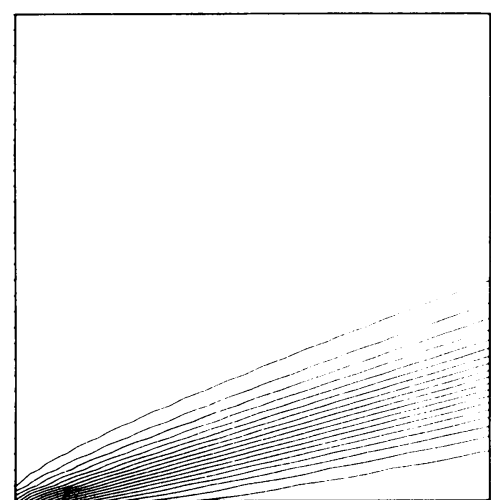

FIGURE 1a

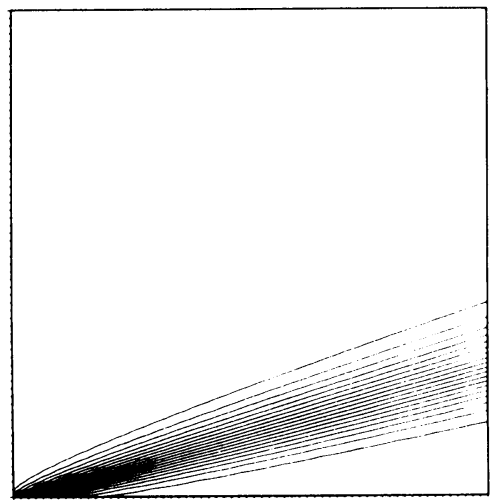

FIGURE 1c

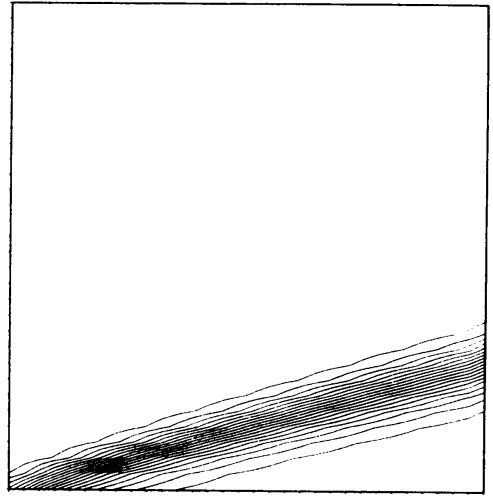

FIGURE 1b

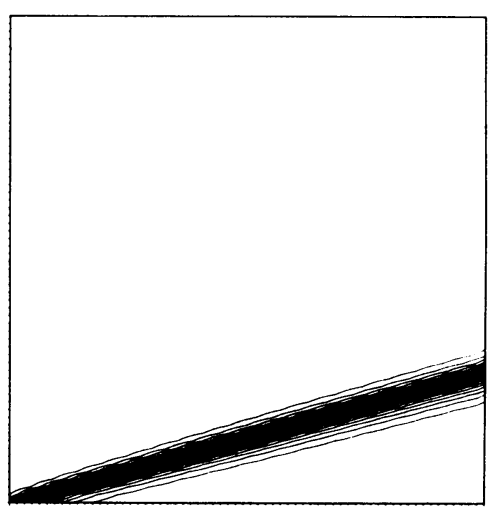

FIGURE 1d

\section{FIGURE 1}

Contour plots of the numerical solution of the first-and second-order scheme, $\phi=15^{\circ}$.

Figures $1 \mathrm{a}$ and $1 \mathrm{~b}$ are solutions on a $32 \times 32$ mesh and Figures $1 \mathrm{c}$ and $1 \mathrm{~d}$ are solutions on a $64 \times 64$ mesh. Figures $1 \mathrm{a}$ and $1 \mathrm{c}$ are obtained with the first-order scheme and Figures $1 \mathrm{~b}$ and $1 \mathrm{~d}$ with the second-order scheme.

From Table 4.1 we conclude that the convergence factors are satisfactory and only weakly dependent of $\phi$. More meshes are needed to estimate the limit values of the convergence factors when $h \downarrow 0$. We have applied the same multigrid strategy for the fully one-sided second-order upwind scheme $(\psi \equiv 1)$. The convergence factors were almost the same as in Table 4.1 (no significant difference). In Figures 1, 2, and 3 we show some numerical solutions. These figures correspond to $\phi=15^{\circ}, 30^{\circ}$ and $45^{\circ}$, respectively.

Example 2. On the rectangle $[-1,1] \times[0,1]$ we consider the linear convection problem

$$
\frac{\partial u}{\partial t}+y \frac{\partial u}{\partial x}-x \frac{\partial u}{\partial y}=0 .
$$

Hence, this problem can be written in the form $(2.1),(2.2)$ with

$$
\begin{gathered}
f^{+}(x, y, u)=y u, \\
g^{+}(x, y, u)=\left\{\begin{array}{ll}
-x u & \text { if } x<0, \\
0 & \text { if } x>0,
\end{array} \quad g^{-}(x, y, u)= \begin{cases}0 & \text { if } x<0, \\
-x u & \text { if } x>0 .\end{cases} \right.
\end{gathered}
$$




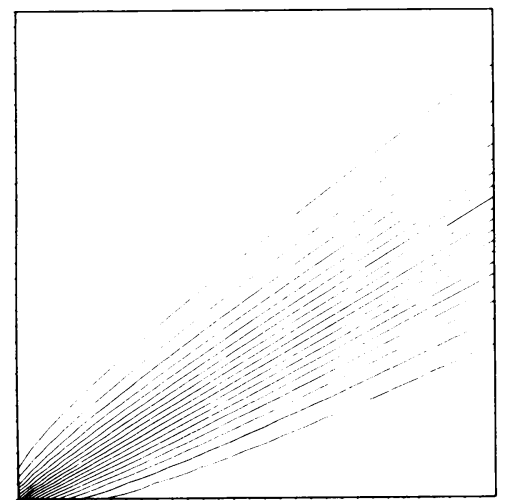

Figure 2a

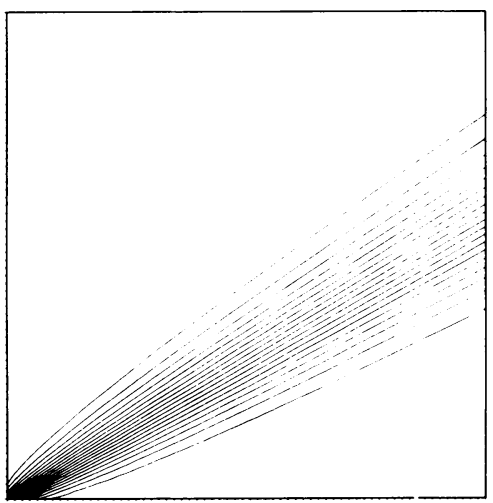

Figure 2c

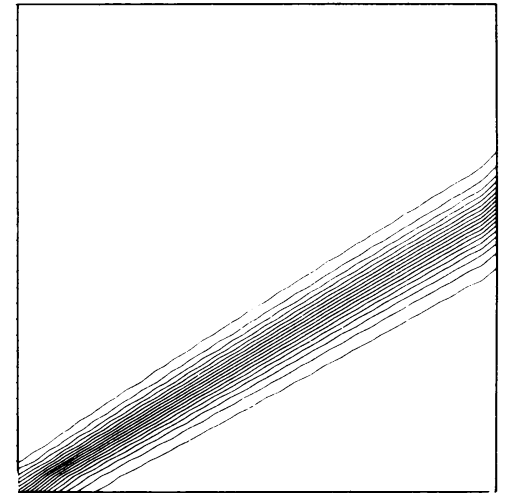

Figure 2b

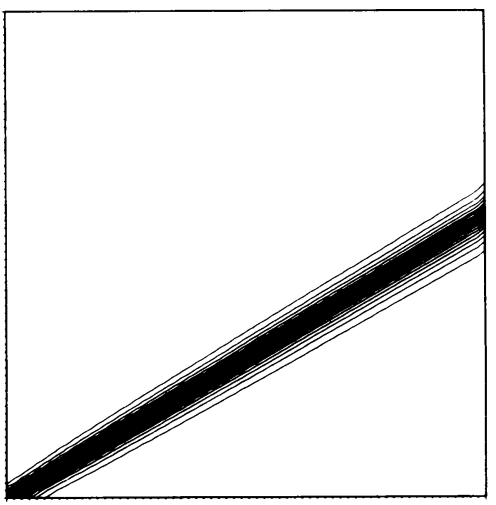

FIGURE 2d

FIGURE 2

As Figure 1 but $\phi=30^{\circ}$.

The boundary conditions (steady-state problem) are

$$
\begin{cases}u(x, 0)=0 & \text { if } x<-0.65 \\ u(x, 0)=1 & \text { if }-0.65<x<-0.35 \\ u(x, 0)=0 & \text { if }-0.35<x<0 \\ u(-1, y)=0 & 0<y<1 \\ u(x, 1)=1 & 0<x<1\end{cases}
$$

The exact solution is

$$
\begin{array}{ll}
u_{e x}(x, y)=1 & \text { if } 0.35<\sqrt{x^{2}+y^{2}}<0.65 \\
u_{e x}(x, y)=0 & \text { otherwise. }
\end{array}
$$

Computations have been carried out on a $32 \times 16$ mesh $(h=1 / 16)$ and on a $64 \times 32$ mesh $(h=1 / 32)$. The observed convergence rates for the multigrid process were 0.29 and 0.44 , respectively. In Figure 4 we show the numerical solution on the $64 \times 32$ mesh. 


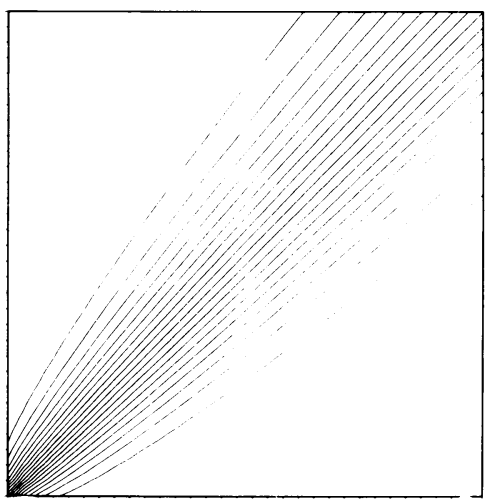

FIgURE 3a

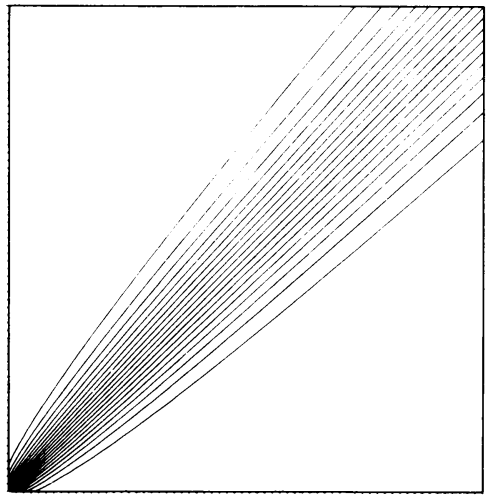

Figure 3c

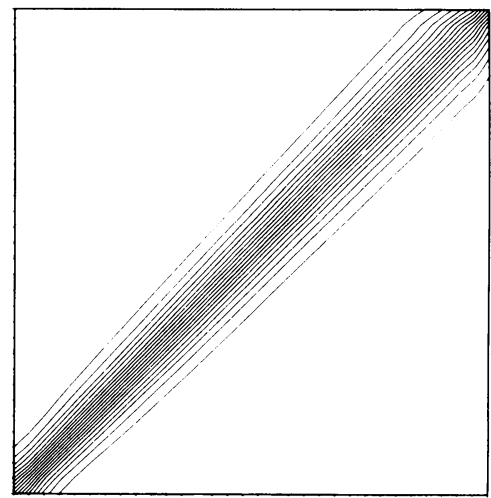

FIGURE 3b

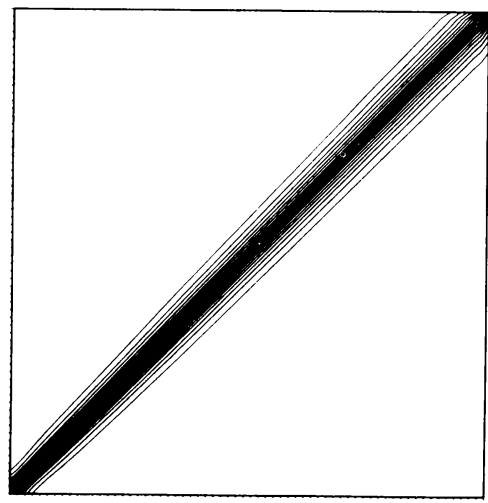

Figure 3d

\section{FIGURE 3}

As Figure 1 but $\phi=45^{\circ}$.

Example 3. On the square $[0,1] \times[0,1]$ we consider the nonlinear problem

$$
\frac{\partial}{\partial t} u+\frac{\partial}{\partial x} f(u)+\frac{\partial}{\partial y} g(u)=0
$$

where

$$
f(u)=\frac{1}{2} u^{2}, \quad g(u)=u \text {. }
$$

Hence,

$$
f^{+}(u)=\frac{1}{2}\left\{u^{+}\right\}^{2}, \quad f^{-}(u)=\frac{1}{2}\left\{u^{-}\right\}^{2}, \quad g^{+}(u)=u, \quad g^{-}(u)=0,
$$

where $u^{+}=\max (u, 0), u^{-}=\min (u, 0)$.

The steady-state equation is

$$
\frac{\partial}{\partial y} u+\frac{\partial}{\partial x} \frac{1}{2} u^{2}=0
$$

i.e., the inviscid Burgers' equation. Two different sets of boundary conditions have been considered. 


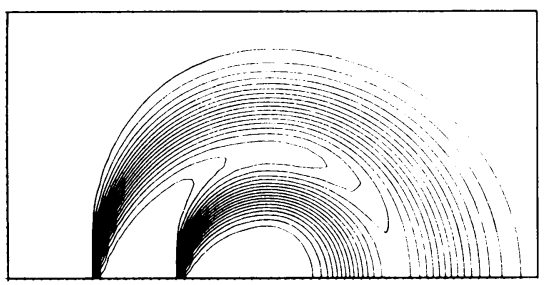

Figure 4a

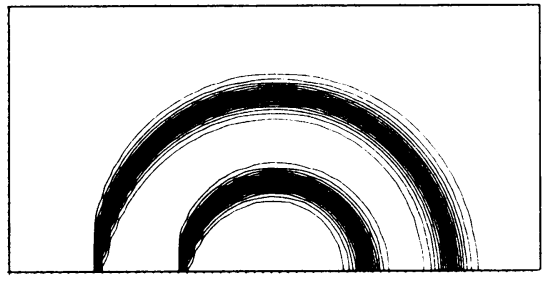

Figure 4b

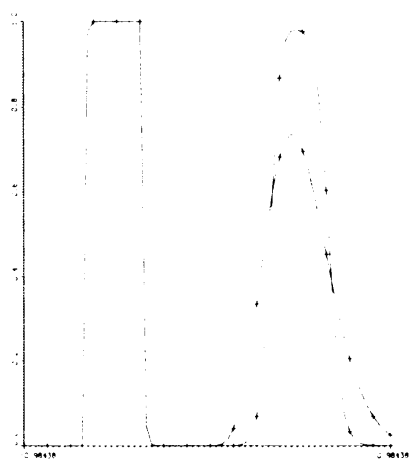

FIGURE 4c

Figure 4

Numerical solutions for Example 2.

A contour plot of the first-order solution is shown in Figure 4a, the second-order solution in $4 \mathrm{~b}$. In Figure $4 \mathrm{c}$ the solutions at the boundary $y=0$ are shown for the first- and second-order scheme.

Problem 3a. With the boundary conditions

$$
\begin{cases}u(0, y)=1, & 0<y<1, \\ u(1, y)=-1, & 0<y<1, \\ u(x, 0)=1-2 x, & 0<x<1,\end{cases}
$$

the solution is (see Figure a)

$$
\begin{array}{ll}
u_{e x}(x, y)=1 & \text { if }(x, y) \text { in region } A, \\
u_{e x}(x, y)=-1 & \text { if }(x, y) \text { in region } B, \\
u_{e x}(x, y)=\frac{1-2 x}{1-2 y} & \text { if }(x, y) \text { in region } C .
\end{array}
$$

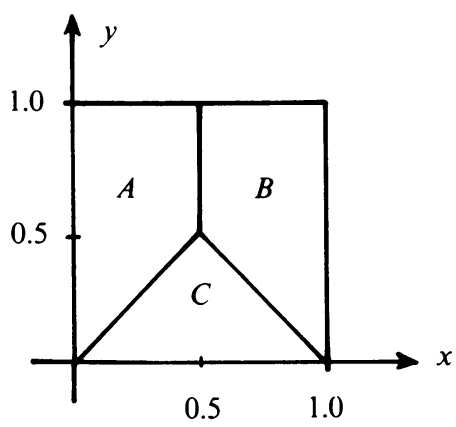

Figure a

The regions $A$ and $B$ are separated by a shock, originating at $(x, y)=(0.5,0.5)$.

Computations have been carried out on a $32 \times 32$ and a $64 \times 64$ grid. The observed convergence factors for the multigrid process were 0.49 and 0.46 , respectively. In Figure 5 we show contour plots of the numerical solutions on the $64 \times 64$ grid. Figure 5a shows the first-order, and Figure $5 \mathrm{~b}$ the second-order solution. 


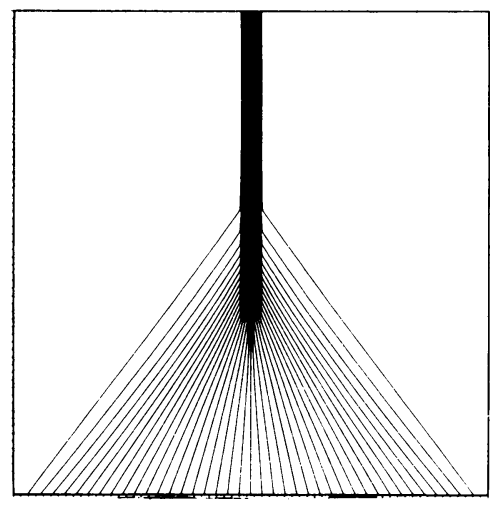

FIGURE 5a

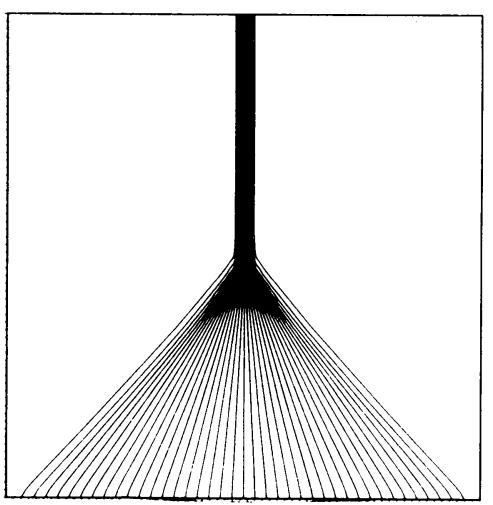

Figure 5b

Figure 5

Contour plots of the first- and second-order solutions of the inviscid Burgers' equation (considered as a boundary value problem) with a shock.

Problem 3b. With the boundary conditions

$$
\begin{cases}u(0, y)=1.5, & 0<y<1 \\ u(1, y)=-0.5, & 0<y<1, \\ u(x, 0)=1.5-2 x, & 0<x<1\end{cases}
$$

the solution is (see Figure b)

$$
\begin{array}{ll}
U_{e x}(x, y)=1.5 & \text { if }(x, y) \text { in region } A, \\
U_{e x}(x, y)=-0.5 & \text { if }(x, y) \text { in region } B, \\
U_{e x}(x, y)=\frac{1.5-2 x}{1-2 y} & \text { if in region } C .
\end{array}
$$

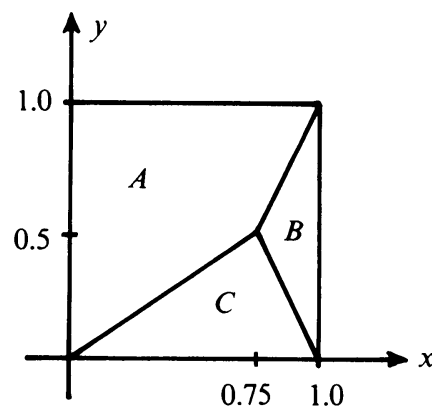

Figure b

Regions $A$ and $B$ are separated by an oblique shock, originating at $(x, y)=$ $(0.75,0.50)$.

Again, computations have been carried out on a $32 \times 32$ and a $64 \times 64$ mesh. The observed convergence factors were 0.35 and 0.45 , respectively. In Figure 6 we show the contour plots of the numerical solutions on the $64 \times 64$ grid. Figure 6 a shows the first-order, and Figure $6 \mathrm{~b}$ the second-order solution. 


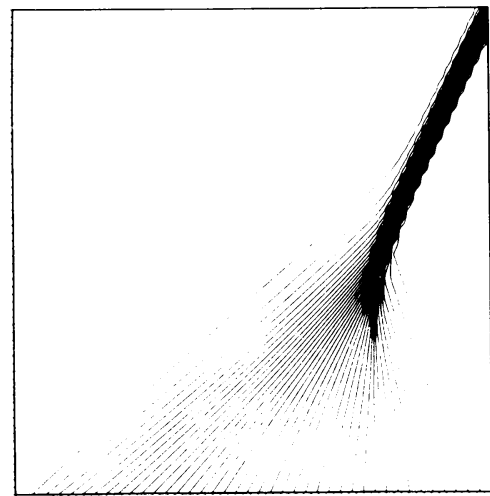

FIGURE 6a

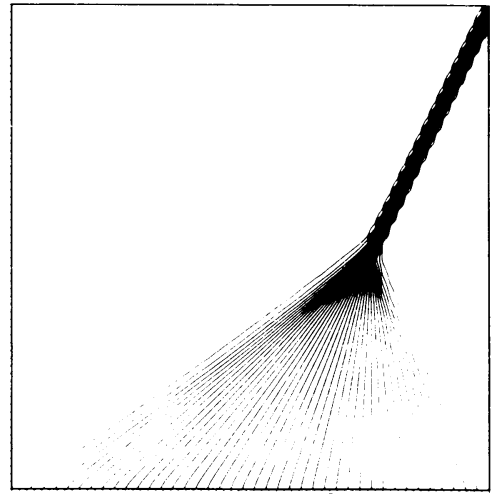

FIGURE $6 b$

\section{FIGURE 6}

As Figure 5 but with an oblique shock.

5. Conclusions. In this paper, it is shown that the multigrid method can be an efficient solution procedure to obtain steady-state solutions of second-order accurate, monotone upwind schemes for hyperbolic conservation laws, also when the solution contains an (oblique) contact discontinuity or shock. The key to success for the multigrid method is the efficient relaxation (smoothing) procedure. It has been shown that a symmetric block Gauss-Seidel underrelaxation (each block is associated with 4 cellis) is an efficient smoothing operator. Furthermore, the coarse-grid operators have been obtained by a Galerkin approximation which has the important practical consequence that coarse-grid operators are first-order accurate. Hence, simple relaxation methods, such as point Gauss-Seidel relaxation, are efficient on the coarser grids.

By the use of a definition of monotonicity, based on positivity of coefficients, it is shown that there is no conflict between second-order accuracy and monotonicity (neither in one nor in more dimensions). The limiter, applied in the second-order scheme to preserve monotonicity is the smooth limiter of van Albada.

The ideas described in this paper can be generalized to systems of hyperbolic conservation laws as, e.g., the Euler equations. A report on this application is in preparation.

Acknowledgment. The author would like to thank P. Hemker, B. van Leer and P. Wesseling for reading the manuscript, and P. de Zeeuw for his assistance in the computational work.

Centre for Mathematics and Computer Science

P. O. Box 4079

1009 AB Amsterdam, The Netherlands

1. A. BrandT, Guide to Multigrid Development (W. Hackbusch and U. Trottenberg, eds.), Multigrid Methods, Lecture Notes in Math., vol. 960, Springer-Verlag, Berlin and New York, 1982, pp. 220-312.

2. S. Chakravarthy \& S. Osher, High Resolution Applications of the Osher Upwind Scheme for the Euler Equations, AIAA paper 83-1943, Proc. AIAA Sixth Computational Fluid Dynamics Conf. (Danvers, Mass., July 1983), pp. 363-372. 
3. J. B. Goodman \& R. J. LeVeque, "On the accuracy of stable schemes for 2D scalar conservation laws," Math. Comp., v. 45, 1985, pp. 15-21.

4. A. Harten, J. M. Hyman \& P. D. Lax, "On finite-difference approximations and entropy conditions for shocks," Comm. Pure Appl. Math., v. 29, 1976, pp. 197-322.

5. A. Harten, "High resolution schemes for hyperbolic conservation laws," J. Comput. Phys., v. 49, 1983, pp. 357-393.

6. P. W. Hemker \& S. P. Spekreisse, "Multiple grid and Osher's scheme for the efficient solution of the steady Euler equations," Appl. Numer. Math., v. 2, 1986, 475-493.

7. P. W. HemKer \& S. P. SPEKREIJSE, Multigrid Solution of the Steady Euler Equations (D. Braess, W. Hackbusch and U. Trottenberg, eds.), Advances in Multigrid Methods, Notes on Numerical Fluid Dynamics, vol. 11, Vieweg Publ. Co., Braunschweig, 1985, pp. 33-44.

8. D. C. Jespersen, "Design and implementation of a multigrid code for the Euler equations," Appl. Math. Comput., v. 13, 1983, pp. 357-374.

9. W. A. Mulder \& B. VAN Leer, Implicit Upwind Methods for the Euler Equations, AIAA paper 83-1930, AIAA 6th Computational Fluid Dynamics Conf. (Danvers, Mass., 1983), pp. 303-310.

10. S. P. SPEKREIJSE, Second Order Accurate Upwind Solutions of the $2 D$ Steady Euler Equations by the Use of a Defect Correction Method, Multigrid Methods II, Proc. 2nd European Multigrid Conference (Cologne, 1985), Lecture Notes in Math., vol. 1228, Springer-Verlag, Berlin and New York, 1985, pp. 285-300.

11. P. K. SWEBy, "High resolution schemes using flux limiters for hyperbolic conservation laws," SIAM J. Numer. Anal., v. 21, 1984, pp. 995-1011.

12. G. D. van Albada, B. van Leer \& W. W. Roberts, JR., "A comparative study of computational methods in cosmic gas dynamics," Astronom. and Astrophys., v. 108, 1982, pp. 76-84.

13. B. VAN LeER, "Towards the ultimate conservative difference scheme II. Monotonicity and conservation combined in a second-order scheme," J. Comput. Phys., v. 14, 1974, pp. 361-370.

14. B. VAN LeER, "Towards the ultimate conservative difference scheme IV. A new approach to numerical convection," J. Comput. Phys., v. 23, 1977, pp. 276-299.

15. B. VAN Leer, Upwind Difference Methods for Aerodynamic Problems Governed by the Euler Equations, Lectures in Appl. Math., vol. 22, Amer. Math. Soc., Providence, R. I., 1985, pp. 327-336. 